\title{
Correction of approximation errors with Random Forests applied to modelling of cloud droplet formation
}

\author{
A. Lipponen ${ }^{1}$, V. Kolehmainen ${ }^{1}$, S. Romakkaniemi ${ }^{1}$, and H. Kokkola ${ }^{2}$ \\ ${ }^{1}$ Department of Applied Physics, University of Eastern Finland, P.O. Box 1627, 70211 Kuopio, Finland \\ ${ }^{2}$ Finnish Meteorological Institute, Kuopio Unit, P.O. Box 1627, 70211 Kuopio, Finland
}

Correspondence to: A. Lipponen (antti.lipponen@uef.fi)

Received: 1 March 2013 - Published in Geosci. Model Dev. Discuss.: 19 April 2013

Revised: 23 October 2013 - Accepted: 30 October 2013 - Published: 16 December 2013

\begin{abstract}
In atmospheric models, due to their computational time or resource limitations, physical processes have to be simulated using reduced (i.e. simplified) models. The use of a reduced model, however, induces errors to the simulation results. These errors are referred to as approximation errors. In this paper, we propose a novel approach to correct these approximation errors. We model the approximation error as an additive noise process in the simulation model and employ the Random Forest (RF) regression algorithm for constructing a computationally low cost predictor for the approximation error. In this way, the overall simulation problem is decomposed into two separate and computationally efficient simulation problems: solution of the reduced model and prediction of the approximation error realisation. The approach is tested for handling approximation errors due to a reduced coarse sectional representation of aerosol size distribution in a cloud droplet formation calculation as well as for compensating the uncertainty caused by the aerosol activation parameterization itself. The results show a significant improvement in the accuracy of the simulation compared to the conventional simulation with a reduced model. The proposed approach is rather general and extension of it to different parameterizations or reduced process models that are coupled to geoscientific models is a straightforward task. Another major benefit of this method is that it can be applied to physical processes that are dependent on a large number of variables making them difficult to be parameterized by traditional methods.
\end{abstract}

\section{Introduction}

In numerical simulations of complicated physical phenomena, one usually has to balance between the model accuracy and the computation time. Reduction in computation time is typically obtained by using reduced models for some of the functions in the model. The use of reduced models, however, result in errors in model output. The errors are referred to as the approximation errors (AE).

In this paper, we consider the approximation errors caused by coarse discretization of aerosol size distributions in sectional aerosol models. In sectional models, the continuous aerosol particle size distributions are represented with discrete size sections (e.g. Weisenstein et al., 2007; Jacobson, 2001; Rodriguez and Dabdub, 2004; Kokkola et al., 2008). The accuracy of the description of the size distribution increases with increasing number of size sections. The computational demand of the model, however, is heavily increased with the number of the sections. Therefore, a compromise between the model accuracy and the computational time has to be made to construct a feasible model for simulations of atmospheric scale.

The main mechanism by which atmospheric aerosol particles affect the climate is by modifying the concentration of cloud condensation nuclei $(\mathrm{CCN})$ followed by changes in cloud droplet number concentration (the indirect effect of aerosols). While it is well known that the number of CCN in the atmosphere has increased, the effect of these additional $\mathrm{CCN}$ on cloud properties is still the largest single source of uncertainty in the current estimates of the anthropogenic radiative forcing (Forster et al., 2007). Thus, solving the cloud activation of the aerosol particles more accurately, 
would reduce the uncertainty in the estimated aerosol indirect effect. Current aerosol-climate models include parameterizations for calculating cloud activation of aerosol that use the above mentioned sectional approach (Abdul-Razzak and Ghan, 2002; Nenes and Seinfeld, 2003). These parameterizations introduce uncertainty in CDNC estimation due to highly simplified description of aerosol activation process. Beyond this, coarse size resolution of the aerosol size distribution that is used as an input for a cloud activation parameterization translate to approximation errors in the calculated aerosol indirect effect.

Recently, an approach for compensating approximation errors in inverse problems was proposed by Kaipio and Somersalo (Kaipio and Somersalo, 2005). The approach is known as the approximation error approach. This far, the approach has mainly been applied to so-called soft field tomography imaging problems that are related to estimation of spatially distributed parameters of partial differential equations from boundary measurements. In such problems, the approach has been successful, for example, in compensation of approximation errors due to coarse finite element discretization (Arridge et al., 2006; Nissinen et al., 2009), unknown nuisance parameters (Nissinen et al., 2009, 2011; Kolehmainen et al., 2011), and the truncation of the computational domain (Lehikoinen et al., 2007; Kolehmainen et al., 2009).

The main idea in the approximation error approach is to model the error between the accurate and approximate computational models as an additive noise process. The realisation of the approximation error noise is obviously unknown and cannot be computed without solving the accurate model and knowing the unknown parameters. However, given the prior probability density models of all the unknowns, the inverse problem can be marginalized over the unknown approximation error in an approximate way by utilising a Gaussian estimate for the joint probability density of the approximation error and the unknown parameters. For a detailed explanation, see Kolehmainen et al. (2011).

In this paper, we propose a novel approach for handling approximation errors in simulation models. The approach is an extension of the approximation error approach. Similarly as in applications of the approximation error approach to inverse problems, the discrepancy between the outputs of accurate and reduced models is modelled as an additive approximation error noise process in the simulation model. However, whereas in the framework of inverse problems the uncertainty related to the approximation errors is taken care of by marginalization, here we propose to construct a computationally low-cost predictor model that computes an estimate for the realisation of the approximation error given in the input parameters and solution of the reduced model. This way the solution of the simulation problem is decomposed into a computationally efficient approximation of solving the reduced computation model and estimating the value of the additive approximation error.
One computationally simple and light-weight and recently widely used function approximation approach is to employ RFs. The RFs are predictive models introduced in Breiman (2001). A RF model consists of an ensemble of binary tree predictors. Each of these tree predictors is trained based on the training data.

The aim of the RF model construction is to get numerous tree models that slightly differ from each other. This is achieved by introducing randomization in the tree construction. The constructed RF models are further used for the function output prediction. The prediction of the RF model is computed by averaging the predictions of each (almost) unbiased tree model in the ensemble. This averaging should therefore increase the accuracy of the RF model over a single tree prediction accuracy. Recently, the RF models have been applied to classification and regression problems including classification of climate zones (Bechtel and Daneke, 2012), earthquake induced damages (Tesfamariam and Liu, 2010), remote-sensing data (Pal, 2005) and disease prediction (Munro et al., 2006; Yao et al., 2013). In papers by Bechtel and Daneke (2012), Tesfamariam and Liu (2010), Pal (2005), a comparison between different algorithms were carried out. Despite its simplicity, the RF was observed to perform at least equally well as the more complicated algorithms in classification and regression problems.

We employ the RF approach for construction of the predictor model for the approximation errors in the simulation model. Here it should be noted that the proposed approach is not restricted to the RFs only and some other type of models, such as neural networks (Rojas, 1996; Haykin, 2009), could have been used as well. The training data for the RF algorithm is a set of approximation error realisations between the accurate and reduced models corresponding to a set of random samples of the input parameters that are sampled from the prior probability density models. The computation of the training data involves solution of the computationally demanding accurate model as many times as the number of samples. This step, however, can be done as precomputation and needs to be carried out only once. Given the trained RF model, the accurate model can then be approximated by the sum of the reduced model and the predicted approximation error in the actual simulations.

The proposed approach is evaluated in the case of cloud droplet number concentration (CDNC) estimation from sectional aerosol particle size distribution using the cloud droplet formation parameterization by Abdul-Razzak and Ghan (2002). We consider the approximation errors caused by using a coarse sectional representation of the aerosol particle size distributions as well as the error caused by using the parameterization of aerosol activation instead of model actually simulating the process of aerosol growth to cloud droplets. The results show that the proposed approach gives a significantly improved accuracy over the conventional way of using the reduced model only with the cost of a small increase in the computational burden. 
The rest of the paper is organised as follows. The approximation error approach and the RF models are explained and the approach for prediction of approximation errors using the RF models is proposed in Sect. 2. In Sect. 3, the cloud droplet formation parameterization by Abdul-Razzak and Ghan (2002) (ARG) and the air parcel model used in the simulations are briefly reviewed. In Sect. 4, the proposed approach is applied and evaluated in cloud droplet formation calculation. The conclusions are given in Sect. 5 .

\section{Correction of approximation errors with Random Forests}

\subsection{Approximation error model}

Let $\boldsymbol{f}(\boldsymbol{x}), \boldsymbol{f}: \mathbb{R}^{N} \rightarrow \mathbb{R}^{M}$ denote the numerically convergent but computationally too time consuming computational model. Here, $x \in \mathbb{R}^{N}$ denotes the inputs of the function. Instead of using the model $\boldsymbol{f}(\boldsymbol{x})$, one wishes to use a computationally low cost reduced model

$\tilde{\boldsymbol{f}}(\tilde{\boldsymbol{x}}), \quad \tilde{\boldsymbol{f}}: \mathbb{R}^{\tilde{N}} \rightarrow \mathbb{R}^{M}, \quad \tilde{N}<N, \quad \tilde{\boldsymbol{x}}=P(\boldsymbol{x})$,

where $P$ is typically a model reduction mapping from higher dimensional space to a lower dimensional space. However, the approximation errors caused by the model reduction can often render the simulation results unreliable, or even useless.

Using the approximation error model (Kaipio and Somersalo, 2005), we write the simulator as

$$
\begin{aligned}
f(\boldsymbol{x}) & =\tilde{\boldsymbol{f}}(\tilde{\boldsymbol{x}})+[\boldsymbol{f}(\boldsymbol{x})-\tilde{\boldsymbol{f}}(\tilde{\boldsymbol{x}})] \\
& =\tilde{\boldsymbol{f}}(\tilde{\boldsymbol{x}})+\boldsymbol{\epsilon}
\end{aligned}
$$

where $\boldsymbol{\epsilon}(\boldsymbol{x})=\boldsymbol{f}(\boldsymbol{x})-\tilde{\boldsymbol{f}}(\tilde{\boldsymbol{x}})$ represents the approximation error. Notice that model (2) is accurate but the exact realisation of the approximation error for a given realisation of input parameters $\boldsymbol{x}$ can only be evaluated by solving the computationally demanding accurate model $\boldsymbol{f}(\boldsymbol{x})$, which we wish to avoid in the first place. In the present work, our objective is to construct a computationally fast predictor model for the realisation of the approximation error

$\tilde{\boldsymbol{g}}(\tilde{\boldsymbol{x}})=\hat{\boldsymbol{\epsilon}}$

where $\hat{\boldsymbol{\epsilon}}$ is the predictor for the approximation error $\boldsymbol{\epsilon}$. With this model, the simulation of $\boldsymbol{f}(\boldsymbol{x})$ can be approximated in a computationally efficient form

$\boldsymbol{f}(\boldsymbol{x}) \approx \tilde{\boldsymbol{f}}(\tilde{\boldsymbol{x}})+\tilde{\boldsymbol{g}}(\tilde{\boldsymbol{x}})$

for a given realisation of the reduced parameterization $\tilde{\boldsymbol{x}}$. For this, we model $(\boldsymbol{x}, \boldsymbol{\epsilon})$ as vector valued random variables and utilise the RF model for the construction of the predictor $\tilde{\boldsymbol{g}}(\tilde{\boldsymbol{x}})$.

\subsection{Simulation of training data for the Random Forest algorithm}

The construction of a predictor model $\tilde{\boldsymbol{g}}(\tilde{\boldsymbol{x}})$ requires a set of feasible realisations of the random variables $\left\{\tilde{\boldsymbol{x}}_{k}, \boldsymbol{\epsilon}_{k}, k=\right.$ $1, \ldots, N\}$. Firstly, this step involves drawing $N$ random realisations of $\boldsymbol{x}_{k}$ from the prior probability density model $\pi(\boldsymbol{x})$, or alternatively, one can utilise set of existing data (e.g. measured realisations of $\boldsymbol{x}$ ) if available. Secondly, one has to compute realisations $\boldsymbol{\epsilon}_{k}=\boldsymbol{f}\left(\boldsymbol{x}_{k}\right)-\tilde{\boldsymbol{f}}\left(P\left(\boldsymbol{x}_{k}\right)\right)$ of the approximation error for each of the samples to obtain the training data $\left\{\tilde{\boldsymbol{x}}_{k}, \boldsymbol{\epsilon}_{k}, k=1, \ldots, N\right\}$. Obviously, this step involves solving the accurate and computationally demanding model $\boldsymbol{f}(\boldsymbol{x}) N$ times. However, this computationally demanding part has to be done only once for the construction of the simulation model (4). This model can then be used to approximate the accurate model $\boldsymbol{f}(\boldsymbol{x})$, for example, within aerosolclimate models where the computational times are a critical issue. The outline of the simulation of the training data is presented in Algorithm 1.

\subsection{Random Forests}

RFs developed by Breiman (2001) are used for classification and regression. The RF algorithm uses training data to construct an RF model used for predicting a class in which the given input belongs (classification) or the output of a function the input would give (regression). An RF model consist of an ensemble of classification or regression trees. Each tree in the RF is grown independently of each other and based on a slightly different training set to avoid overfitting of the model. In particular, each training set is obtained as a random subset of the original training set. Further, the reason for constructing an ensemble of tree models, not a single tree model, is to increase the accuracy and reduce the uncertainty of the overall prediction. In this paper, the RF models for regression are used for the construction of the predictor $\tilde{\boldsymbol{g}}(\tilde{\boldsymbol{x}})$.

In case of regression, the RF model consists of an ensemble of regression tree models. A regression tree model is a sequence of rules that is used for function output prediction with given inputs. The sequence of rules forms a binary tree structure and it is evaluated by following the nodes starting from the uppermost node referred to as the root node. Each node rule consists of a pair of input variable index and split threshold value. In the node evaluation, the value of the input variable indicated by the index is compared with the split threshold value. If the input data variable value is less than the threshold value the left branch of the node is followed. In other cases, the right branch is followed. The tree structure is followed until a node that has no child nodes is reached. These nodes are referred to as the leaf nodes. The tree model output prediction is selected as the output value indicated by the leaf node. Finally, the output of the RF model is computed as the average of all the individual tree model outputs. For an illustrative example of a regression tree, see Fig. 1. 


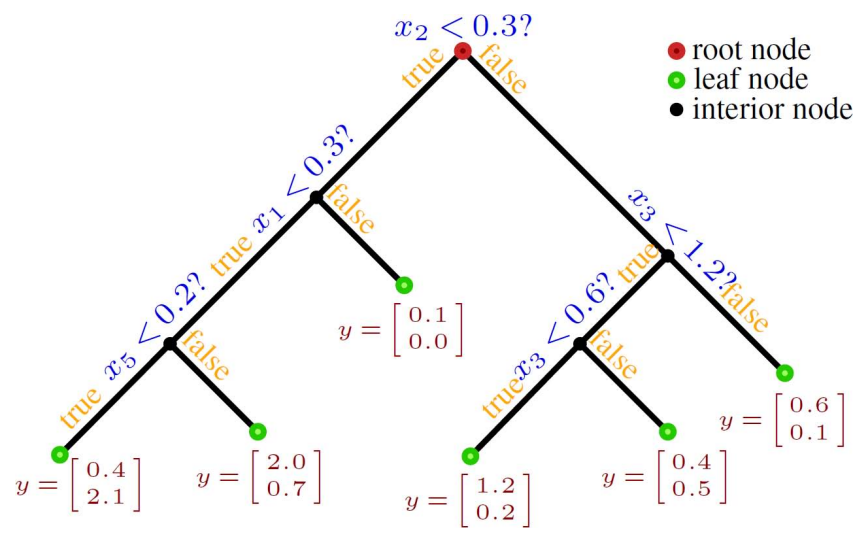

Fig. 1. An illustrative example of a regression tree.

As stated above, an ensemble of trees is constructed from the training data $\left\{\tilde{\boldsymbol{x}}_{k}, \boldsymbol{\epsilon}_{k}\right\}$. The samples $\tilde{\boldsymbol{x}}_{k}$ and $\boldsymbol{\epsilon}_{k}$ are considered as the inputs and outputs of the function, respectively, which the RF model to be constructed is approximating. The training procedure of an RF is carried out as follows. First, random samples from the training data are selected and assigned to the root node of a regression tree. Typically, the number of selected samples is the same as the number of samples in the original training data. The random selection is carried out with replacement and therefore the samples from the original training dataset are not necessary selected or may be selected multiple times. Second, a random subset of the input variables is selected and all possible splits of the training data samples with respect to these variables are tested. The split that minimises mean squared error of the regression tree is selected and the training data samples are assigned to the new child nodes according to the selected split rule. This splitting is carried out as long as nodes with enough samples assigned to them exist. This procedure of training regression trees is repeated until the predeterminated number trees are trained. A more detailed description on training an RF model is presented, for example, in the paper by Breiman (2001).

In this paper, we use an open source machine learning library scikit-learn ${ }^{1}$ for Python (Pedregosa et al., 2011) to implement the RF. In the scikit-learn library, the RF used for regression is named as RandomForestRegressor. In this paper, we study the effect of three different RandomForestRegressor training parameters on the RF accuracy. These three parameters are $n \_$estimators, the number of trees in the forest, max_features, the number of input variables to consider when looking for the best split, and min_samples_split, the minimum number of training samples required to split a node. For all the other RandomForestRegressor parameters, the default values are used. An example code listing for training and evaluating the RF with the scikit-learn is given in Fig. 2.

\footnotetext{
1 version 0.14 .1 , http://scikit-learn.org/
}

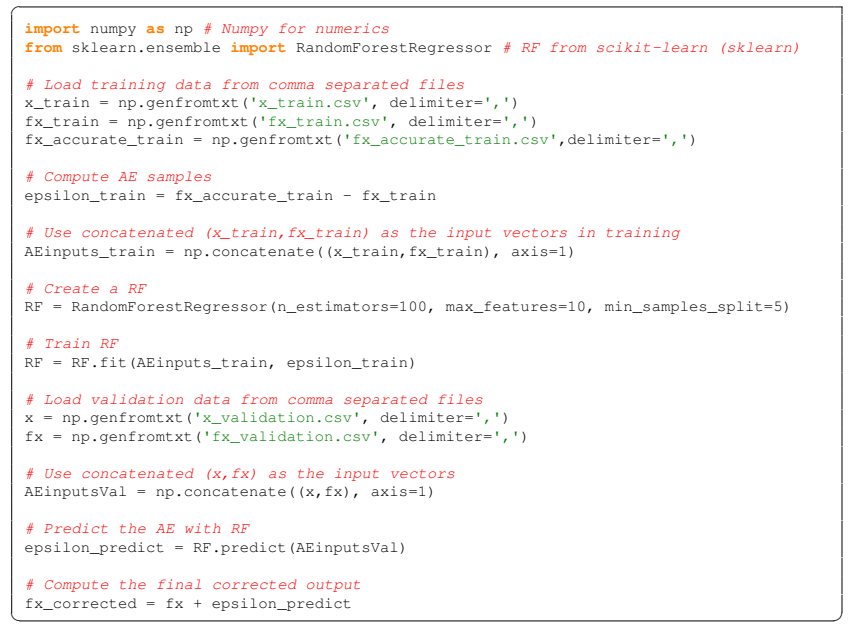

Fig. 2. An example code listing for training and evaluating an RF model in scikit-learn.

\section{Cloud droplet formation parameterization}

Formation of cloud droplets in the atmosphere is a dynamical process affected by local meteorology and aerosol particles acting as cloud condensation nuclei. In the most sophisticated parameterizations, CDNC is calculated based on aerosol particle size distribution and chemical composition, pressure, temperature and vertical velocity of air parcel forming the cloud (Abdul-Razzak et al., 1998; Abdul-Razzak and Ghan, 2000, 2002; Nenes and Seinfeld, 2003; Fountoukis and Nenes, 2005).

The simulations in this study are conducted using the SALSA sectional aerosol model developed for atmospheric models (Kokkola et al., 2008; Bergman et al., 2012). In SALSA, aerosol size distribution is divided to different subranges based on the particle size $(3-50,50-700$, and 700$10000 \mathrm{~nm})$. The size resolution differs between the subranges depending on how sensitive the aerosol processes are to particle sizes of given subrange. In this study, the size sections within subranges have a constant volume ratio between the adjacent sections. When using the default setup of SALSA, it has 10 size sections divided so that there are 3 sections in the first subrange, 4 in the second subrange, and 3 in the third subrange. A more detailed description of the model is given by Kokkola et al. (2008).

SALSA includes all relevant microphysical processes such as condensation of sulfate and organic carbon, nucleation of new particles, hydration, and coagulation. However, in this study we are interested in the effect of the size resolution on the calculated number of cloud droplets, and the SALSA is used only to create aerosol size distribution and to calculate the CDNC using the ARG parameterization. It has to be noted that here we refer to CDNC as the cloud droplet number concentration at the cloud base. The activation parameterization by Abdul-Razzak and Ghan (2002) does not 
Table 1. The numbers and names of the input variables in the ARG parameterizations.

\begin{tabular}{lccc}
\hline Parameterization input variable name & $\begin{array}{c}\text { Number of variables } \\
\text { in 4 size sections } \\
\text { parameterization }\end{array}$ & $\begin{array}{c}\text { Number of variables } \\
\text { 7 size sections } \\
\text { parameterization }\end{array}$ & $\begin{array}{c}\text { Number of variables } \\
\text { 70 size sections } \\
\text { parameterization }\end{array}$ \\
\hline Temperature & 1 & 1 & 1 \\
Pressure & 1 & 1 & 1 \\
Vertical velocity & 1 & 1 & 1 \\
Particle number concentration & 8 & 13 & 130 \\
Volume concentration of sulphate & 8 & 13 & 130 \\
Volume concentration of organic carbon & 2 & 4 & 40 \\
Volume concentration of dust & 8 & 13 & 130 \\
\hline Total number of variables & 29 & 46 & 433 \\
\hline
\end{tabular}

take into account CDNC affecting processes within cloud, e.g. entrainment and as such is not a complete representation of CDNC. Also, we are omitting the first subrange as usually the cloud droplet nucleation in the atmosphere is not affected by these particles as they are too small to act as cloud condensation nuclei. For simplicity, in this study we have also assumed that aerosol is composed of only one highly hygroscopic compound (sulphate), one slightly hygroscopic compound (organic carbon) and one non-hygroscopic compound (dust).

Beyond evaluating the size resolution effect, we also study if the RF can be used to minimise the parameterization errors caused by the ARG parameterization itself in the estimation of CDNC. For that purpose we use an air parcel model, that solves the differential equations describing the aerosol growth to cloud droplets by water uptake in an adiabatically ascending air parcel. The model used has been described in detail elsewhere (Kokkola et al., 2003) and it has been used in several aerosol cloud interaction studies (e.g. Romakkaniemi et al., 2005, 2012). In the model, the differential equations are solved using an ordinary differential equation solver DLSODE (www.netlib.org), which solves initial-value problems for stiff or non-stiff ordinary differential equations using backward differentiation formulae. The liquid phase thermodynamics needed for the vapour pressures on the liquid particle surfaces are calculated with AIM, which is a chemical equilibrium code (Clegg et al., 1998). The aerosol size distribution is represented by the method of moving sections, with 250 sections in this study. In the current study the model is used in its simplest setup, where only the condensation of water is taken into account without other microphysical processes.

\section{Models, simulations and results}

\subsection{Accurate and reduced models}

Let $f(\boldsymbol{x}) \in \mathbb{R}$ denote the numerically convergent computational ARG cloud droplet formation parameterization that
Table 2. Size section configurations of the cloud droplet formation parameterizations used in simulations.

\begin{tabular}{ccc}
\hline $\begin{array}{c}\text { Total number } \\
\text { of size sections } \\
\text { in the model }\end{array}$ & $\begin{array}{c}\text { Size sections in the } \\
\text { diameter range } \\
50-700 \mathrm{~nm}\end{array}$ & $\begin{array}{c}\text { Size sections in the } \\
\text { diameter range } \\
0.7-10 \mu \mathrm{m}\end{array}$ \\
\hline 70 & 40 & 30 \\
7 & 4 & 3 \\
4 & 2 & 2 \\
\hline
\end{tabular}

computes the value of the CDNC for the given input $x$. By numerically convergent, it is meant that the output of the parameterization do not significantly change if more size sections were added. The input parameter vector $\boldsymbol{x}$ contains aerosol particle size and composition distributions, vertical velocity, pressures and temperature information. For the names and number of input variables in different parameterizations see Table 1. In the following computations, the number of size sections for the representation of the particle size distributions is 70 , see Table 2 . With this discretization, the average simulation time of the accurate model is about $0.92 \mathrm{~ms}$.

In the parameter vector $\tilde{\boldsymbol{x}}$ of the reduced model $\tilde{f}(\tilde{\boldsymbol{x}})$, the number of size sections for the aerosol particle size distributions have been significantly reduced. We consider two different levels of model reduction. In the first one, the number of size sections is 7 and in the second one 4 , see Table 2 . The average computation times are about 0.11 and $0.07 \mathrm{~ms}$ for the 7 and 4 sections parameterizations, respectively. Thus, when reducing from 70 size sections to 7 or 4 sections the average reductions in computation times are about 89 and $93 \%$, respectively.

\subsection{Construction of the RF predictor model}

The size of sample set $\left\{\boldsymbol{x}_{k}\right\}$ was selected as $N=50000$ for the construction of the training data (Algorithm 1). The realisations $\left\{\boldsymbol{x}_{k}\right\}$ of the input parameters were drawn from their 
Table 3. The prior probability distribution models used for the cloud droplet formation parameterization inputs. The $\mathcal{U}, \mathcal{N}$, and $\Gamma$ denote the uniform, Gaussian and gamma distributions, respectively. The details of the probability distribution functions are shown in Table 4.

\begin{tabular}{lll}
\hline Variable & Distribution & Unit \\
\hline$w$ & $\Gamma(1.25,0.75)$ & $\mathrm{ms}^{-1}$ \\
$p$ & $\mathcal{U}(10000,100000)$ & $\mathrm{P}$ \\
$T$ & $\mathcal{U}(240,300)$ & $\mathrm{K}$ \\
$n_{\text {tot }, 1}$ & $\Gamma(2,800)$ & $\mathrm{cm}^{-3}$ \\
$\mu_{1}$ & $\mathcal{U}(50,80)$ & $\mathrm{nm}$ \\
$\sigma_{1}$ & $\mathcal{N}(1.5,0.125)$ & \\
$n_{\text {tot }, 2}$ & $\Gamma(3,200)$ & $\mathrm{cm}^{-3}$ \\
$\mu_{2}$ & $\mathcal{U}(100,200)$ & $\mathrm{nm}^{-3}$ \\
$\sigma_{2}$ & $\mathcal{N}(1.5,0.125)$ & \\
$n_{\text {tot }, 3}$ & $\Gamma(1.25,0.75)$ & $\mathrm{cm}^{-3}$ \\
$\mu_{3}$ & $\mathcal{U}(500,1500)$ & $\mathrm{nm}$ \\
$\sigma_{3}$ & $\mathcal{N}(1.5,0.125)$ & \\
\hline
\end{tabular}

prior probability distribution models, which were selected so that the realisations are plausible representations of their values in the nature. The aerosol particle number distribution $n=n(d)$, where $d$ is the diameter of the particle, was modelled as a sum of three log-normal modes representing the Aitken, accumulation and coarse mode aerosols:

$n(d)=\sum_{i=1}^{3} n_{i}(d)$

where each of the modes was modelled by

$n_{i}(d)=\frac{n_{\mathrm{tot}, i}}{d \sqrt{2 \pi\left(\log \left(\sigma_{i}\right)\right)^{2}}} \exp \left\{-\left(\log \left(d / \mu_{i}\right)\right)^{2} /\left(2 \sigma_{i}^{2}\right)\right\}$

where the $n_{\text {tot, } i}$ is the total number of particles in mode $i$, and $\sigma_{i}$ and $\mu_{i}$ the shape and $\log$-scale parameters of mode $i$. The parameters of the prior probability distribution models used in the generation of the vertical velocity $w$, pressure $p$, temperature $T$, and the particle number distribution parameter $n_{i}, \sigma_{i}, \mu_{i}$ samples are shown in Table 3 and the respective probability density functions are shown in Table 4 . The histograms of the temperature, pressure and vertical velocity samples, and the particle number distribution parameters in the training sample set $\left\{\boldsymbol{x}_{k}\right\}$ are shown in Figs. 3 and 4, respectively. The aerosol particle volume size distributions were constructed with the particle number distributions of the modes and randomly distributed volume fractions of each compound. The volume fractions for the sulphate were drawn from an uniform distribution $\mathcal{U}(0.01,1)$ separately for each mode. Further, the fractions of dust and organic carbon were drawn from uniform distributions such that the sum of the compound fractions was 1 .
Table 4. The notations used for the probability distributions and their probability density functions. $\Gamma(k)$ denotes the Gamma function.

\begin{tabular}{ll}
\hline Notation & Probability density function $\pi(x)$ \\
\hline$x \sim \mathcal{U}(a, b)$ & $\begin{cases}\frac{1}{b-a}, & a \leq x \leq b \\
0, & \text { otherwise }\end{cases}$ \\
$x \sim \mathcal{N}\left(\bar{x}, \sigma^{2}\right)$ & $\frac{1}{\sigma \sqrt{2 \pi}} \exp \left(-\frac{(x-\bar{x})^{2}}{2 \sigma^{2}}\right)$ \\
$x \sim \Gamma(k, \theta)$ & $\frac{1}{\Gamma(k) \theta^{k}} x^{k-1} \exp \left(-\frac{x}{\theta}\right)$ \\
\hline
\end{tabular}
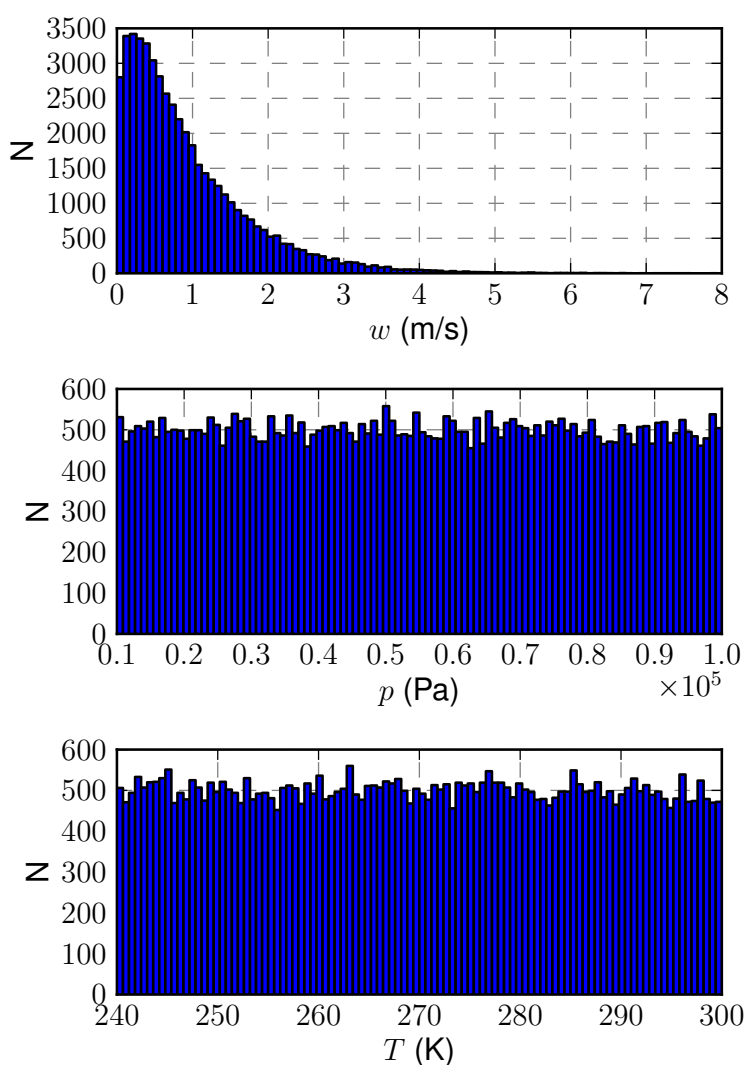

Fig. 3. Histograms of vertical velocity $w$, pressure $p$ and temperature $T$ in the sample set used for constructing the approximation error samples. $N$ denotes the number of samples.

Figure 5 shows the output values of the accurate parameterization against the output of the approximate parameterization for the set of training samples $\left\{\boldsymbol{x}_{k}\right\}$. In the top panel, the reduced model uses 4 size sections for the size distributions and in the bottom 7 size sections. The black line shows the identity line $f(\boldsymbol{x})=\tilde{f}(\tilde{\boldsymbol{x}})$ corresponding to the case that accurate and reduced models match. The average relative errors in the CDNC values were 20.4 and $54.6 \%$ for the 7 and 4 size sections parameterizations, respectively. The reason for the 

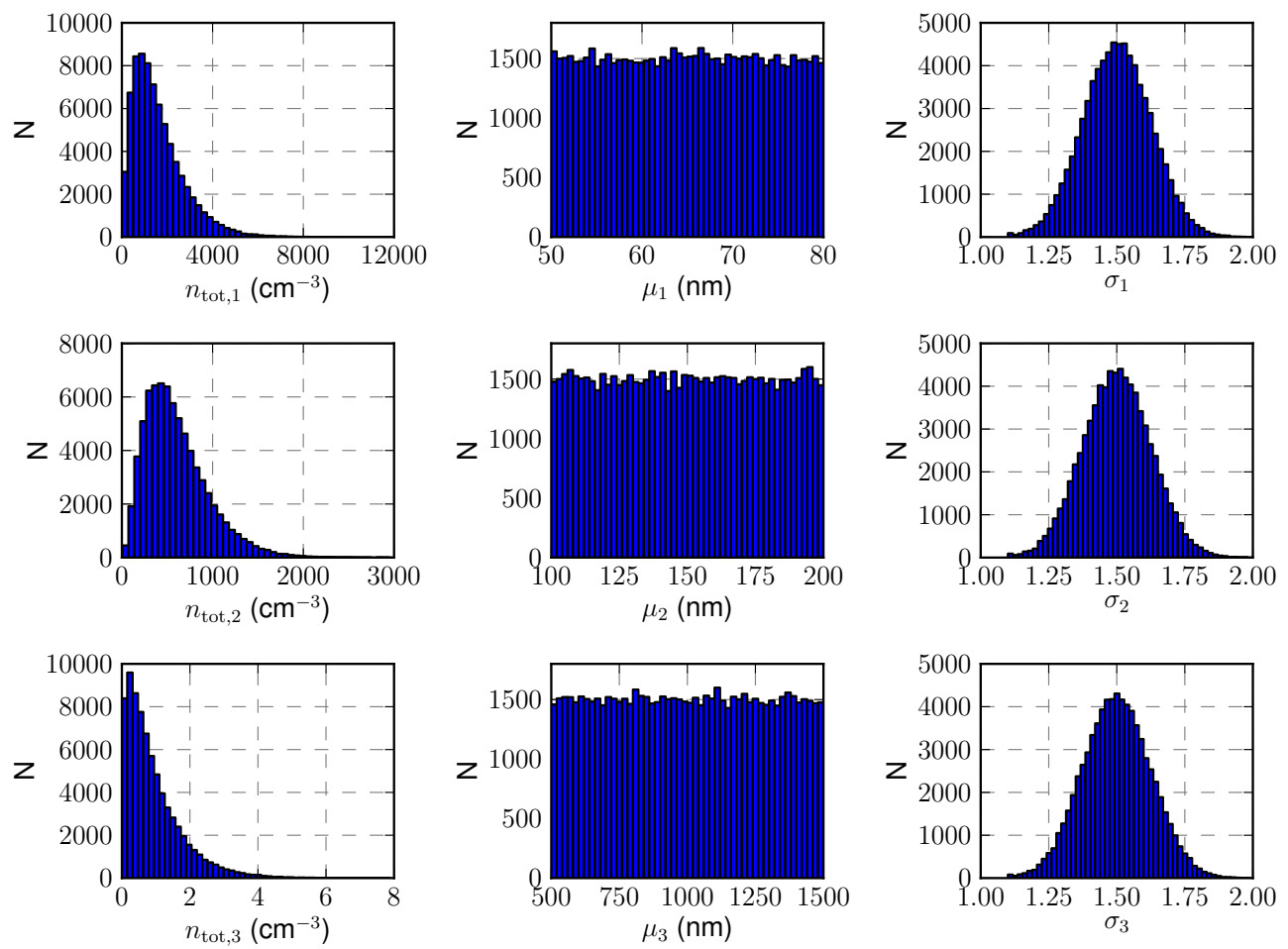

Fig. 4. Histograms for number concentrations of particles $n_{i}$, scale parameters $\mu_{i}$ and shape parameters $\sigma_{i}$ for the log-normal modes $i=$ 1,2,3. $N$ denotes the number of samples.

(a)

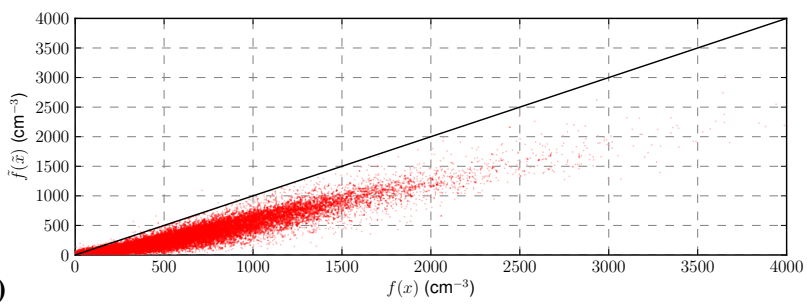

(b)

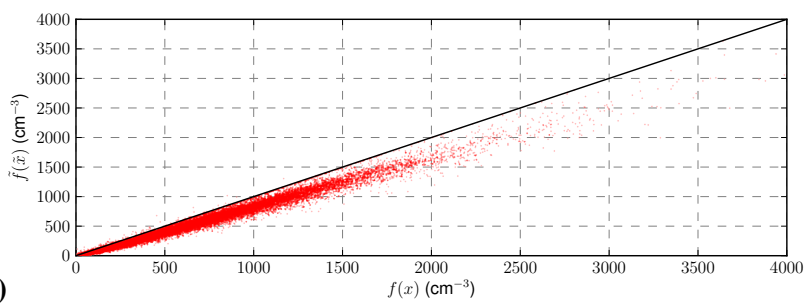

Fig. 5. Cloud droplet number concentrations (CDNC) computed with the approximate model $\tilde{f}(\tilde{\boldsymbol{x}})$ as functions of CDNCs given by the accurate model $f(\boldsymbol{x})$. (a) Approximate parameterization with 4 size sections for the aerosol particle size distributions. (b) Approximate parameterization with 7 size sections for the aerosol particle size distributions. Black solid lines represent the identity lines.

lower CDNC with the smaller number of size sections is the lower maximum supersaturation when using the ARG parameterization.
Given the samples $\left\{\boldsymbol{x}_{k}\right\}$, the realisations of the approximation error were simulated as

$\left\{\epsilon_{k}=f^{\prime}\left(\boldsymbol{x}_{k}\right)-\tilde{f}^{\prime}\left(P\left(\boldsymbol{x}_{k}\right)\right), k=1, \ldots, N\right\}$.

where $\quad f^{\prime}\left(x_{k}\right)=\log \left(f\left(x_{k}\right)\right) \quad$ and $\quad \tilde{f}^{\prime}\left(P\left(x_{k}\right)\right)=$ $\log \left(\tilde{f}\left(P\left(\boldsymbol{x}_{k}\right)\right)\right)$. It was found that the use of linear or logarithmic scale for the CDNC in the RF training resulted in similar root-mean-square errors and bias in the estimates. In some RF models, however, the mean relative error was more than ten times higher with the linear scale than with the logarithmic scale. Therefore, we chose to use CDNC with logarithmic scale in the computations. The histograms of the approximation errors $\epsilon$ for both the 7 and 4 size sections parameterizations are shown in Fig. 6.

Finally, the sample sets $\left\{\boldsymbol{x}_{k}, \tilde{f}^{\prime}\left(P\left(\boldsymbol{x}_{k}\right)\right)\right\}$ and $\left\{\epsilon_{k}\right\}$ were used as the RF training set inputs and outputs, respectively, and the RF models were trained as described in the Sect. 2.3. Also here, the addition of logarithms of the coarse parameterization outputs in the training set slightly improved the RF model accuracy and was therefore used. Once the RF predictor $\tilde{g}$ was constructed, the output of the accurate simulator $f(\boldsymbol{x})$ was approximated with

$$
f(\boldsymbol{x}) \approx \exp (\log (\tilde{f}(\tilde{\boldsymbol{x}}))+\tilde{g}(\tilde{\boldsymbol{x}}, \tilde{f}(\tilde{\boldsymbol{x}}))) .
$$


(a)
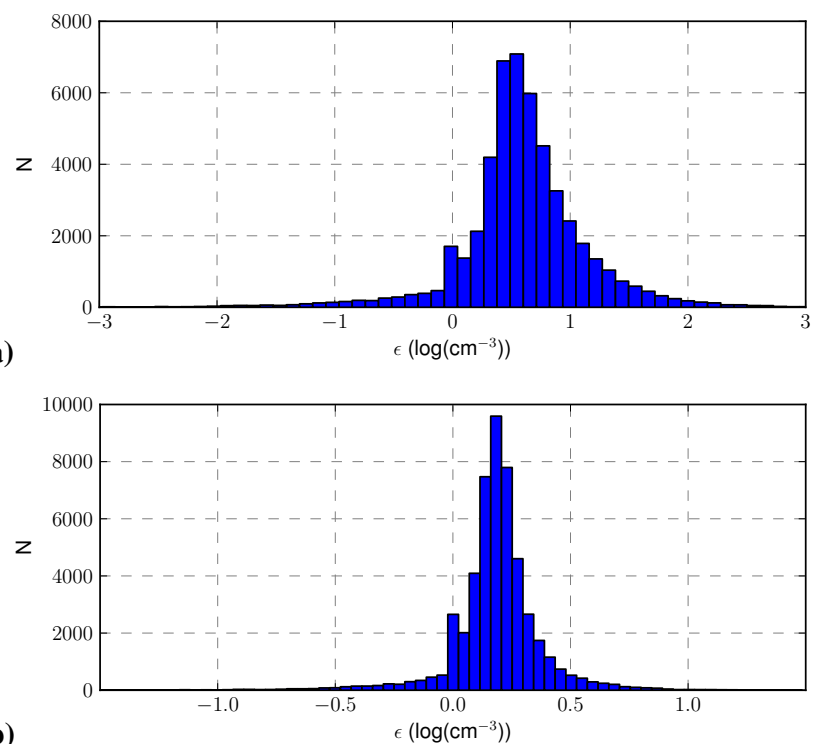

(b)

Fig. 6. Histograms of the approximation errors $\epsilon(\boldsymbol{x}) . N$ denotes the number of samples. (a) Approximate parameterization with 4 size sections for the aerosol particle size distributions. (b) Approximate parameterization with 7 size sections for the aerosol particle size distributions.

\subsection{Results}

\subsubsection{Compensation of approximation errors due to reduced coarse sectional representation of the aerosol size distribution}

To evaluate the proposed approach, multiple RF predictor models for the approximation errors corresponding to both approximate ARG parameterizations, with 7 and 4 size sections, were constructed with different RF training parameters. All possible combinations of parameter sets $\{25,50,100,200,400\},\{5,10,15,25\}$, and $\{2,5,15,25,100\}$ for $n \_$estimators, max_features and min_samples_split, respectively, were used. These parameter ranges were selected based on a test which showed that selecting values outside these ranges either resulted in poor model accuracy or considerably larger computational burden with no significant improvement on the model accuracy. To avoid overoptimistic results, the constructed AE models were evaluated with a separate validation set of 25000 samples of ARG model inputs. The validation set was sampled similarly as the training set but the samples were not included in the training of the RF model.

All predictor models were evaluated using the validation set, and the root-mean-squared error (RMSE) $\epsilon_{\mathrm{RMSE}}$ and mean relative error (MRE) $\epsilon_{\mathrm{MRE}}$ estimates were computed.
The error estimates were computed as

$$
\begin{aligned}
\epsilon_{\mathrm{RMSE}} & =\sqrt{\frac{1}{N} \sum_{i=1}^{N}\left(f\left(\boldsymbol{x}_{i}\right)-\hat{f}\left(\tilde{\boldsymbol{x}}_{i}\right)\right)^{2}}, \\
\hat{f}(\tilde{\boldsymbol{x}}) & =\exp (\log (\tilde{f}(\tilde{\boldsymbol{x}}))+\tilde{g}(\tilde{\boldsymbol{x}}, \tilde{f}(\tilde{\boldsymbol{x}})))
\end{aligned}
$$

and

$\epsilon_{\mathrm{MRE}}=\frac{1}{N} \sum_{i=1}^{N} \frac{\left|f\left(\boldsymbol{x}_{i}\right)-\hat{f}\left(\tilde{\boldsymbol{x}}_{i}\right)\right|}{\left|f\left(\boldsymbol{x}_{i}\right)\right|}$.

As the construction of an RF model is random, the tests were repeated 25 times for each AE model to also evaluate the random variations in the results. The average RMSE and MRE, average computation time of the approximation error model, and the model parameters for both of the approximate parameterizations $\tilde{f}(\tilde{\boldsymbol{x}})$ corresponding to 20 different combinations of RF training parameters (n_estimators, max_features, and min_samples_split) are given in Table 5 for the parameterization with 7 size sections and Table 6 for the parameterization with 4 size sections. Complete results tables are given in the supplementary material of the paper. The bottom row in both Tables gives the respective errors between the accurate parameterization $f(\boldsymbol{x})$ and reduced parameterization $\tilde{f}(\tilde{\boldsymbol{x}})$ without approximation error correction. The CDNC values computed with the accurate parameterization $f\left(\boldsymbol{x}_{j}\right)$ as a function of the AE corrected CDNC values using the predictor $\tilde{g}$ with the lowest RMSE error are shown in Fig. 7. Panel a shows the case for the reduced model with 4 size sections and panel $b$ the case with 7 size sections for the particle size distributions.

The results show that by using the AE correction with the RF predictor model, both the RMSE and MRE errors are significantly decreased. In the case of the reduced parameterization $\tilde{f}(\tilde{\boldsymbol{x}})$ with 7 size sections, the RF training parameter selections $n \_$estimators $=400$, max_features $=2$, and min_samples_split $=25$ resulted in the overall model in which both the RMSE and MRE were the smallest. Here, the approximation error correction decreased the RMSE and the MRE to values less than 30 and $50 \%$, respectively, of the RMSE and MRE values of the CDNC computed without the approximation error correction. In the case of the reduced parameterization $\tilde{f}(\tilde{\boldsymbol{x}})$ with 4 size sections, the lowest RMSE was obtained with the RF training parameters $n \_$estimators $=400$, max_features $=2$, and min_samples_split $=15$. Also here, both the RMSE and MRE errors were significantly decreased. Notice that the RMSE errors of the 4 size sections parameterization with the approximation error correction are smaller than the MSE errors of the uncorrected 7 size sections parameterization. Because the RF method is asymptotically unbiased, biases are significantly reduced. For the 4 size sections case, the approximative model (Fig. 5a) has a bias of $260 \mathrm{~cm}^{-3}$, while the AE corrected model's (Fig. 7a) bias is $-0.7 \mathrm{~cm}^{-3}$. For the 7 size sections case, the approximative model and $\mathrm{AE}$ corrected model biases are 97 and $0.4 \mathrm{~cm}^{-3}$, respectively. 
Table 5. Training parameters and results of the AE correction in the case of 7 size sections parameterization: number of trees in the RF model n_estimators, the RF training parameters min_samples_split, and max_features, the mean values of rootmean-squared errors $\epsilon_{\mathrm{RMSE}}$ (RMSE) and mean relative errors $\epsilon_{\mathrm{MRE}}$ (MRE), and the average time used for evaluating the RF model $t$.

\begin{tabular}{|c|c|c|c|c|c|}
\hline 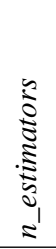 & 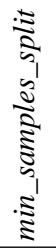 & $\frac{3}{3}$ & 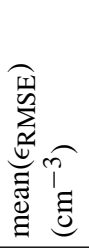 & 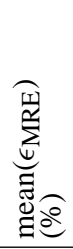 & 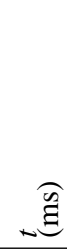 \\
\hline 400 & 2 & 25 & 41.3 & 9.3 & 0.48 \\
\hline 200 & 2 & 25 & 41.4 & 9.3 & 0.27 \\
\hline 400 & 5 & 25 & 41.4 & 9.3 & 0.27 \\
\hline 200 & 5 & 25 & 41.5 & 9.3 & 0.14 \\
\hline 100 & 2 & 25 & 41.8 & 9.4 & 0.14 \\
\hline 100 & 5 & 25 & 41.9 & 9.4 & 0.08 \\
\hline 50 & 5 & 25 & 42.1 & 9.4 & 0.05 \\
\hline 50 & 2 & 25 & 42.1 & 9.4 & 0.09 \\
\hline 400 & 15 & 25 & 42.2 & 9.4 & 0.16 \\
\hline 200 & 15 & 25 & 42.2 & 9.5 & 0.08 \\
\hline 100 & 15 & 25 & 42.4 & 9.5 & 0.05 \\
\hline 50 & 15 & 25 & 42.8 & 9.6 & 0.03 \\
\hline 400 & 25 & 25 & 42.9 & 9.6 & 0.14 \\
\hline 200 & 25 & 25 & 43.0 & 9.6 & 0.07 \\
\hline 100 & 25 & 25 & 43.2 & 9.6 & 0.05 \\
\hline 25 & 5 & 25 & 43.2 & 9.6 & 0.03 \\
\hline 50 & 25 & 25 & 43.4 & 9.7 & 0.03 \\
\hline 400 & 2 & 15 & 43.4 & 9.7 & 0.48 \\
\hline 25 & 15 & 25 & 43.5 & 9.6 & 0.02 \\
\hline 400 & 5 & 15 & 43.5 & 9.7 & 0.28 \\
\hline \multicolumn{5}{|c|}{7 size sections parameterization } & 0.10 \\
\hline
\end{tabular}

The mean of the CDNC values computed with the 70 size sections parameterization: $574.8 \mathrm{~cm}^{-3}$.

The standard deviation of the CDNC values computed with the

70 size sections parameterization: $509.5 \mathrm{~cm}^{-3}$.
Table 6. Training parameters and results of the AE correction in the case of 4 size sections parameterization: number of trees in the RF model n_estimators, the RF training parameters min_samples_split and max_features, the mean values of root-mean-squared errors $\epsilon_{\mathrm{RMSE}}$ (RMSE) and mean relative errors $\epsilon_{\mathrm{MRE}}$ (MRE), and the average time used for evaluating the RF model $t$.

\begin{tabular}{|c|c|c|c|c|c|}
\hline $\begin{array}{l}n \\
\vdots \\
\vdots \\
\vdots \\
\vdots \\
\vdots \\
\vdots \\
0 \\
= \\
\text { a }\end{array}$ & 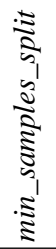 & 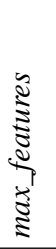 & 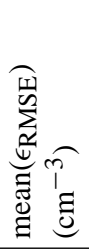 & 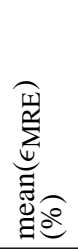 & $\widehat{\mathscr{E}}$ \\
\hline 400 & 2 & 15 & 95.5 & 23.5 & 0.48 \\
\hline 400 & 5 & 15 & 95.7 & 23.5 & 0.26 \\
\hline 200 & 2 & 15 & 95.9 & 23.6 & 0.26 \\
\hline 200 & 5 & 15 & 96.1 & 23.5 & 0.13 \\
\hline 400 & 2 & 25 & 96.7 & 23.1 & 0.49 \\
\hline 100 & 2 & 15 & 96.8 & 23.7 & 0.14 \\
\hline 100 & 5 & 15 & 96.9 & 23.7 & 0.08 \\
\hline 200 & 2 & 25 & 97.2 & 23.2 & 0.26 \\
\hline 100 & 2 & 25 & 97.3 & 23.3 & 0.14 \\
\hline 200 & 5 & 25 & 97.9 & 23.2 & 0.13 \\
\hline 50 & 5 & 15 & 98.2 & 23.8 & 0.05 \\
\hline 50 & 2 & 15 & 98.4 & 24.0 & 0.08 \\
\hline 400 & 5 & 25 & 98.6 & 23.1 & 0.26 \\
\hline 400 & 15 & 15 & 98.8 & 24.0 & 0.16 \\
\hline 200 & 15 & 15 & 99.2 & 24.1 & 0.08 \\
\hline 100 & 5 & 25 & 99.4 & 23.3 & 0.08 \\
\hline 50 & 5 & 25 & 99.6 & 23.5 & 0.05 \\
\hline 100 & 15 & 15 & 99.8 & 24.2 & 0.05 \\
\hline 400 & 2 & 10 & 99.8 & 24.6 & 0.48 \\
\hline 200 & 2 & 10 & 100.1 & 24.6 & 0.26 \\
\hline \multicolumn{6}{|c|}{4 size sections parameterization } \\
\hline
\end{tabular}

The mean of the CDNC values computed with the 70 size sections parameterization: $574.8 \mathrm{~cm}^{-3}$.

The standard deviation of the CDNC values computed with the 70 size sections parameterization: $509.5 \mathrm{~cm}^{-3}$.
The results also show that the RF model training parameters did not significantly affect the accuracy of the $\mathrm{AE}$ model. The RF training parameter affecting the accuracy of the model most was max_features. The randomness in the RF model training caused only minor variations in the resulting RF models showing the robustness of the approach. As an example, in the 7 size sections AE corrected model with the RF training parameters $n \_$estimators $=400$, min_samples_split $=2$, and max_features $=25$, the RMSE and the MRE varied between values $41.2-41.4 \mathrm{~cm}^{-3}$ and $9.23-9.28 \%$, respectively.

The average times to simulate the AE models varied between 0.02 and $0.48 \mathrm{~ms}$ in the case of 7 size sections parameterization and between 0.02 and $0.49 \mathrm{~ms}$ in 4 size sections parameterization on a standard desktop computer. The average time to simulate the reduced model $\tilde{f}(\tilde{\boldsymbol{x}})$ with 7 and 4 size sections were about 0.10 and $0.06 \mathrm{~ms}$, respectively. These $\mathrm{AE}$ model running times for computing $\hat{f}(\tilde{\boldsymbol{x}})$ resulted in overall average runtimes of $0.12-0.58 \mathrm{~ms}$ for the 7 size sections and $0.08-0.54 \mathrm{~ms}$ for the 4 size sections AE corrected parameterizations. Thus, the reduction in computation times of the approximation error corrected models $\hat{f}(\tilde{\boldsymbol{x}})$ is in the range of $37-91 \%$ compared to the run time of the accurate model $f(\boldsymbol{x})$ that was about $0.92 \mathrm{~ms}$. Note that the errors using the fastest RF predictor models are only slightly larger (less than 2 and $0.5 \%$ in the MRE error in cases of 4 and 7 size sections, respectively) compared to the slowest RF model. By using the RF models with the fastest running time, one would still get the RMSE less than $32 \%$ of the RMSE in the reduced models $\tilde{f}(\tilde{\boldsymbol{x}})$ with an increment of computation time from 0.10 to $0.12 \mathrm{~ms}$ for the 7 size sections model and from 0.06 to $0.08 \mathrm{~ms}$ for the 4 sections model. Note that the use 
(a)

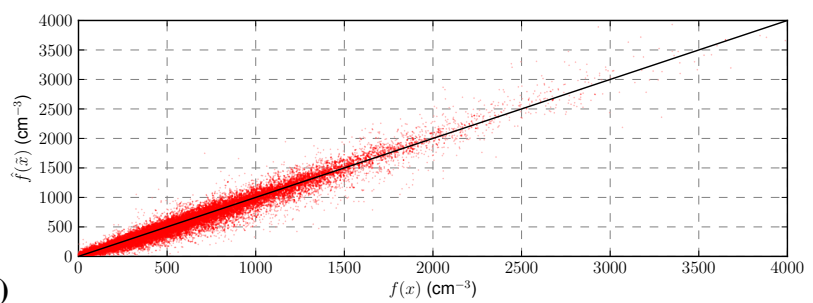

(b)

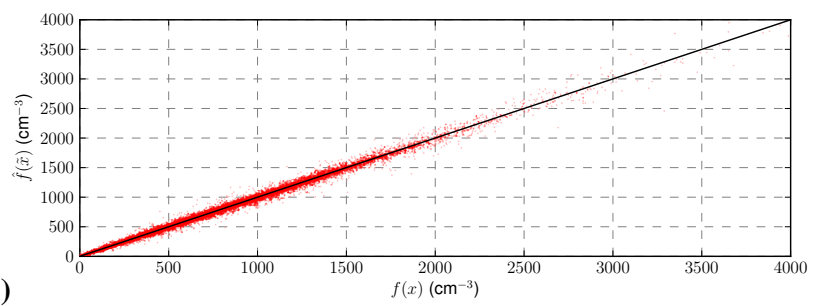

Fig. 7. Cloud droplet number concentrations (CDNC) computed with the approximation error corrected parameterization $\hat{f}\left(\tilde{\boldsymbol{x}}_{j}\right)$ as functions of CDNCs given by the accurate parameterization $f\left(\boldsymbol{x}_{j}\right)$. (a) Reduced parameterization $\tilde{f}(\tilde{\boldsymbol{x}})$ with 4 size sections for the representation of the aerosol particle size distributions. (b) Reduced parameterization $\tilde{f}(\tilde{\boldsymbol{x}})$ with 7 size sections for the representation of the aerosol particle size distributions. Black solid lines represent the identity lines.

of, for example, the RF predictor model with the training parameters $n \_$estimators $=25$, min_samples_split $=15$, and max_features $=25$ resulted in the overall model with only slightly larger (about $0.3 \%$ ) MRE error and $0.46 \mathrm{~ms}$ faster running time compared to the RF model with the smallest MRE error in the case of 7 size sections parameterization. Notice that the computation time of the error prediction by the RF model is independent of the computation times of $f$ or $\tilde{f}$. Thus, the relative time saving by the proposed approach will increase as the computation time of $f$ increases.

\subsubsection{Compensation of approximation errors due to reduction of process model internal numerics}

In addition to the coarse sectional representation of the the aerosol particle distribution as the approximation error source, also simulations with an air parcel model $h\left(\boldsymbol{x}_{j}\right)$ as the accurate model were carried out (see Sect. 3 for the description of $h\left(\boldsymbol{x}_{j}\right)$ ). In these simulations, the 7 size sections ARG parameterization was used as the approximative model. The same training and validation datasets as in the previous ARG parameterization simulations were used and an RF model with parameters $n \_$estimators $=400$, min_samples_split $=$ 2, and max_features $=25$ was trained to predict the approximation errors. The CDNC values computed with and without the approximation error correction as functions of CDNC values of the air parcel model $h\left(\boldsymbol{x}_{j}\right)$ are shown in Fig. 8 . With the approximation error correction, the decrease of the errors was significant, the RMSE decreased from 206.5 to $93.3 \mathrm{~cm}^{-3}$ (the mean of the CDNC values computed with (a)

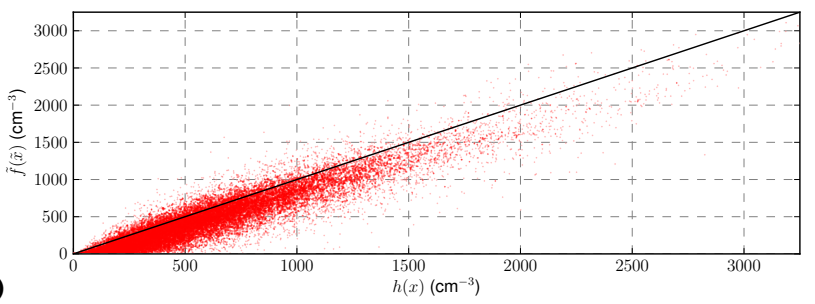

(b)

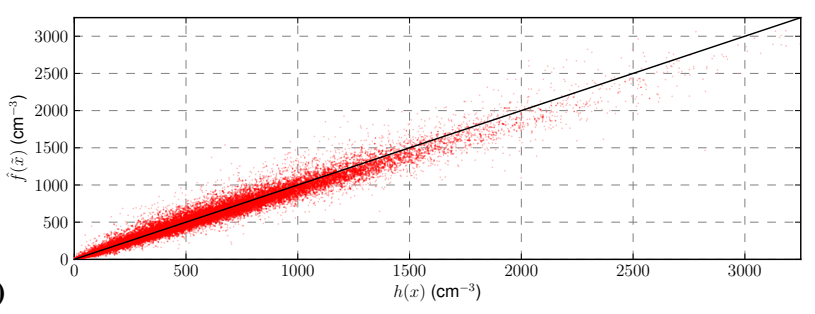

Fig. 8. Cloud droplet number concentrations (CDNC) computed with the the approximate model $\tilde{f}(\tilde{\boldsymbol{x}})$ (a) and the approximation error corrected parameterization $\hat{f}(\tilde{\boldsymbol{x}})$ (b) as functions of CDNCs given by the air parcel model $h\left(\boldsymbol{x}_{j}\right)$. Black solid lines represent the identity lines.

$h\left(\boldsymbol{x}_{j}\right)$ was $\left.452.7 \mathrm{~cm}^{-3}\right)$ and the MRE from 36.6 to $12.6 \%$. The biases in the approximative model (Fig. 8a) and the AE corrected model (Fig. 8b) are 132 and $9.1 \mathrm{~cm}^{-3}$, respectively. These results show that the proposed AE compensation approach is also capable of compensating the errors due to reduction of the internal numerics of the process model.

\section{Conclusions}

Due to computational time and resource limitations related to atmospheric models, several physical processes have to be simulated using reduced models. The use of a reduced model, however, induces approximation errors to the simulation results. In this study, we presented a novel approach to correct these approximation errors and applied it to the calculation of cloud droplet number concentration (CDNC). In the paper, the approximation errors (in CDNC) caused by coarse sectional representation of the aerosol particle distribution and the approximative ARG parameterization of aerosol activation were studied.

In our approach, the approximation errors caused by model reduction are modelled as an additive approximation error noise process in the simulation model and the RF algorithm is utilised for construction of a predictor for the realisation of the approximation error for given model input parameters. This way the accurate simulation model can be approximated in a computationally fast form by evaluating the reduced model and the prediction of the approximation error.

It was found out both in the case of the ARG parameterization and the air parcel model that the RF approach gives 
significantly smaller errors in the CDNC calculation than using the reduced model alone with a small increment in the computational cost. Also the systematic errors caused by reduced model accuracy can be efficiently eliminated. Further it was noted that the use of CDNC in logarithmic scale in the RF training may have high impact on the MRE of the final CDNC estimates. In some cases, the MRE was more than ten times higher if the linear scale for the CDNC was used in the training instead of logarithmic scale.

Another significant result in this study was that if the number of size sections were further decreased from 7 to 4 , the RMS errors in the RF corrected CDNC of the 4 sections model were lower than the errors of the uncorrected 7 sections model. This shows that the RF method could be useful in reducing the number of size distribution parameters, when aerosol models are developed for simulations of decades or centuries. As the method is in no way limited to sectional approach, it could be applied for reducing number of modes in modal models. This type of model reduction has been considered, for example, in Liu et al. (2012).

Here the RF method was employed in the calculation of CDNC with variables typical to atmospheric models. The method can be easily and efficiently extended to take account more complex aerosol including for example surface active (Sorjamaa et al., 2004) or semi-volatile aerosol compounds (Romakkaniemi et al., 2005) by simply adding new variables to the training data. The method is highly efficient especially in the case of physical processes, which have been found to be difficult to parameterize with traditional methods due to high dependence of the processes on several parameters. For example in our simplest case in the calculation of cloud droplet formation, the number of parameters was 29 and thus finding for example analytic formulas for corrections are difficult. A possible topic of future studies is to test the proposed approach with some variant of the RF algorithm, such as the weighted RF (Chen et al., 2004). Further, the proposed approach is rather general and extension of it to different physical simulation models is a straightforward task.

\section{Appendix A}

\section{ALGORITHMS}

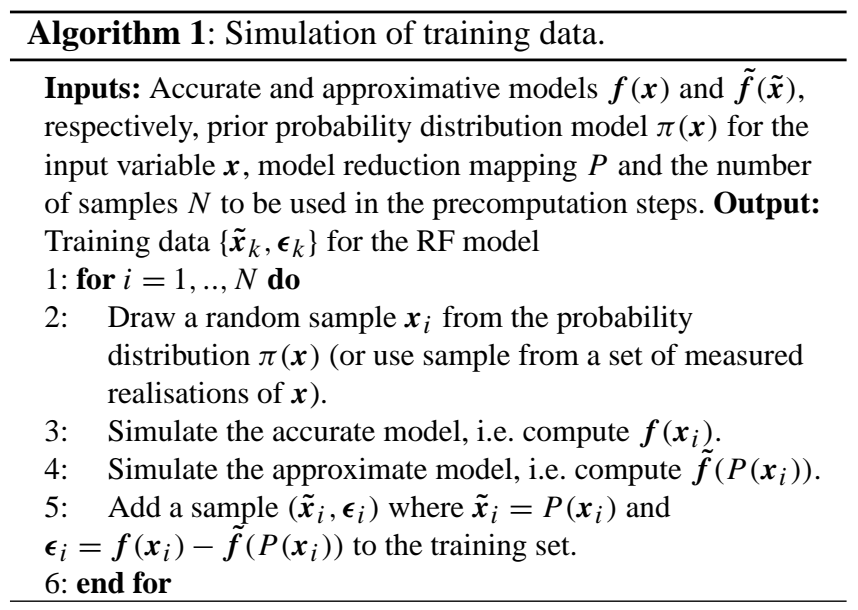

\section{Supplementary material related to this article is available online at http://www.geosci-model-dev.net/6/ 2087/2013/gmd-6-2087-2013-supplement.pdf.}

Acknowledgements. The financial support by the Academy of Finland (project 119270 and Centre of Excellence programmes 1118615 and 250215) and by the strategic funding of the University of Eastern Finland are gratefully acknowledged.

Edited by: A. Kerkweg

\section{References}

Abdul-Razzak, H., Ghan, S. J., and Rivera-Carpio, C.: A parameterization of aerosol activation 1 . single aerosol type, J. Geophys. Res., 103, 6123-6131, doi:10.1029/97JD03735, 1998.

Abdul-Razzak, H. and Ghan, S. J.: A parameterization of aerosol activation 2. Multiple aerosol types, J. Geophys. Res., 105, 68376844, doi:10.1029/1999JD901161, 2000.

Abdul-Razzak, H. and Ghan, S. J.: A parameterization of aerosol activation 3. Sectional representation, J. Geophys. Res., 107, AAC 1-1-AAC 1-6, doi:10.1029/2001JD000483, 2002.

Arridge, S., Kaipio, J., Kolehmainen, V., Schweiger, M. Somersalo, E., Tarvainen, T., and Vauhkonen, M.: Approximation errors and model reduction with an application in optical diffusion tomography, Inverse Probl., 22, 175-195, doi:10.1088/0266-5611/22/1/010, 2006.

Bechtel, B. and Daneke, C.: Classification of local climate zones based on multiple earth observation data, IEEE J. Sel. Top. Appl., 5, 1191-1202, doi:10.1109/JSTARS.2012.2189873, 2012.

Bergman, T., Kerminen, V.-M., Korhonen, H., Lehtinen, K. J., Makkonen, R., Arola, A., Mielonen, T., Romakkaniemi, S., Kulmala, M., and Kokkola, H.: Evaluation of the sectional aerosol microphysics module SALSA implementation in 
ECHAM5-HAM aerosol-climate model, Geosci. Model Dev., 5, 845-868, doi:10.5194/gmd-5-845-2012, 2012.

Breiman, L.: Random Forests, Mach. Learn., 45, 5-32, doi:10.1023/A:1010933404324, 2001.

Chen, C., Liaw, A., and Breiman, L.: Using random forests to learn imbalanced data, Technical Report 666, Statistics Department of University of California, Berkeley, USA, 2004.

Clegg, S. L., Brimblecombe, P., and Wexler, A. S.: Thermodynamical model of the system $\mathrm{H}^{+}-\mathrm{NH}_{4}^{+}-\mathrm{SO}_{4}^{2-}-\mathrm{NO}_{3}^{-}-\mathrm{H}_{2} \mathrm{O}$ at tropospheric temperatures, J. Phys. Chem. A, 102, 2137-2154, 1998.

Forster, P., Ramaswamy, V., Artaxo, P., Berntsen, T., Betts, R., Fahey, D., Haywood, J., Lean, J., Lowe, D., Myhre, G., Nganga, J., Prinn, R., Raga, G., Schulz, M., and Van Dorland, R.: Changes in atmospheric constituents and in radiative forcing, in: Climate Change 2007: The Physical Science Basis, Contribution of Working Group I to the Fourth Assessment Report of the Intergovernmental Panel on Climate Change, edited by: Solomon, S., Qin, D., Manning, M., Chen, Z., Marquis, M., Averyt, K., Tignor, M., and Miller, H., Cambridge University Press, Cambridge, UK and New York, NY, USA, 2007.

Fountoukis, C. and Nenes, A.: Continued development of a cloud formation parameterization for global climate models, J. Geophys. Res., 110, D11212, doi:10.1029/2004JD005591, 2005.

Haykin, S. O.: Neural networks and learning machines, Prentice Hall, New York, 2009.

Jacobson, M. Z.: GATOR-GCMM: a global through urban scale air pollution and weather forecast model. 1. Model design and treatment of subgrid soil, vegetation, roads, rooftops, water, sea ice, and snow, J. Geophys. Res., 106, 5385-5402, doi:10.1029/2000JD900560, 2001.

Kaipio, J. and Somersalo, E.: Statistical and Computational Inverse Problems, Springer, New York, 2005.

Kokkola, H., Romakkaniemi, S., Kulmala, M., and Laaksonen, A.: A cloud microphysics model including trace gas condensation and sulfate chemistry, Boreal Environ. Res., 8, 413-424,2003.

Kokkola, H., Korhonen, H., Lehtinen, K. E. J., Makkonen, R., Asmi, A., Järvenoja, S., Anttila, T., Partanen, A.-I., Kulmala, M., Järvinen, H., Laaksonen, A., and Kerminen, V.-M.: SALSA a Sectional Aerosol module for Large Scale Applications, Atmos. Chem. Phys., 8, 2469-2483, doi:10.5194/acp-8-2469-2008, 2008.

Kolehmainen, V., Schweiger, M., Nissilä, I., Tarvainen, T., Arridge, S., and Kaipio, J.: Approximation errors and model reduction in three-dimensional diffuse optical tomography, J. Opt. Soc. Am. A, 10, 2257-2267, doi:10.1364/JOSAA.26.002257, 2009.

Kolehmainen, V., Tarvainen, T., Arridge, S., and Kaipio, J.: Marginalization of uninteresting distributed parameters in inverse problems - application to diffuse optical tomography, International Journal for Uncertainty Quantification, 1, 1-17, doi:10.1615/Int.J.UncertaintyQuantification.v1.i1.10, 2011.

Lehikoinen, A., Finsterle, S., Voutilainen, A., Heikkinen, L., Vauhkonen, M., and Kaipio, J.: Approximation errors and truncation of computational domains with application to geophysical tomography, Inverse Probl. Imag., 1, 371-389, doi:10.3934/ipi.2007.1.371, 2007.

Liu, X., Easter, R. C., Ghan, S. J., Zaveri, R., Rasch, P., Shi, X., Lamarque, J.-F., Gettelman, A., Morrison, H., Vitt, F., Conley, A., Park, S., Neale, R., Hannay, C., Ekman, A. M. L., Hess, P., Mahowald, N., Collins, W., Iacono, M. J., Brether- ton, C. S., Flanner, M. G., and Mitchell, D.: Toward a minimal representation of aerosols in climate models: description and evaluation in the Community Atmosphere Model CAM5, Geosci. Model Dev., 5, 709-739, doi:10.5194/gmd-5-709-2012, 2012.

Munro, N. P., Cairns, D. A., Clarke, P., Rogers, M., Stanley, A. J., Barrett, J. H., Harnden, P., Thompson, D., Eardley, I., Banks, R. E., and Knowles, M. A.: Urinary biomarker profiling in transitional cell carcinoma, Int. J. Cancer, 119, 2642-2650, 2006.

Nenes, A. and Seinfeld, J.: Parameterization of cloud dropletformation in global climate models, J. Geophys. Res., 108, 4415, doi:10.1029/2002JD002911, 2003.

Nissinen, A., Heikkinen, L., Kolehmainen, V., and Kaipio, J.: Compensation of errors due to discretization, domain truncation and unknown contact impedances in electrical impedance tomography, Meas. Sci. Technol., 20, 105504, doi:10.1088/09570233/20/10/105504, 2009.

Nissinen, A., Kolehmainen, V., and Kaipio, J.: Compensation of modelling errors due to unknown domain boundary in electrical impedance tomography, IEEE T. Med. Imaging, 30, 231-242, 2011.

Pal, M.: Random Forest classifier for remote sensing classification, Int. J. Remote Sens., 26, 217-222, doi:10.1080/01431160412331269698, 2005.

Pedregosa, F., Varoquaux, G., Gramfort, A., Michel, V., Thirion, B., Grisel, O, Blondel, M., Prettenhofer, P., Weiss, R., Dubourg, V., Vanderplas, J., Passos, A., Cournapeau, D., Brucher, M., Perrot, M., and Duchesnay, E.: Scikit-learn: Machine Learning in Python, J. Mach. Learn. Res., 12, 2825-2830, 2011.

Rodriguez, M. and Dabdub, D. J.: IMAGES-SCAPE2: A modeling study of size and chemically resolved aerosol thermodynamics in a global chemical transport model, J. Geophys. Res., 109, D02203, doi:10.1029/2003JD003639, 2004.

Rojas, R.: Neural Networks: A Systematic Introduction, SpringerVerlag, Berlin, 1996.

Romakkaniemi, S., Kokkola, H., and Laaksonen, A.: Parameterization of the nitric acid effect on CCN activation, Atmos. Chem. Phys., 5, 879-885, doi:10.5194/acp-5-879-2005, 2005.

Romakkaniemi, S., Arola, A., Kokkola, H., Birmili, W., Tuch, T., Kerminen, V.-M., Rïsänen, P., Smith, J. N., Korhonen, H., and Laaksonen, A.: Effect of aerosol size distribution changes on AOD, CCN and cloud droplet concentration: Case studies from Erfurt and Melpitz, Germany, J. Geophys. Res., in press, doi:10.1029/2011JD017091, 2012.

Sorjamaa, R., Svenningsson, B., Raatikainen, T., Henning, S., Bilde, M., and Laaksonen, A.: The role of surfactants in Köhler theory reconsidered, Atmos. Chem. Phys., 4, 2107-2117, doi:10.5194/acp-4-2107-2004, 2004.

Tesfamariam, S. and Liu, Z.: Earthquake induced damage classification for reinforced concrete buildings, Struct. Saf., 32, 154-164, doi:10.1016/j.strusafe.2009.10.002, 2010.

Weisenstein, D. K., Penner, J. E., Herzog, M., and Liu, X.: Global 2D intercomparison of sectional and modal aerosol modules, Atmos. Chem. Phys., 7, 2339-2355, doi:10.5194/acp-7-2339-2007, 2007.

Yao, D., Yang, J., and Zhan, X.: A novel method for disease prediction: hybrid of Random Forest and multivariate adaptive regression splines, J. Computers, 8, 170-177, doi:10.4304/jcp.8.1.170177, 2013. 